\title{
Addendum: Multi-scale predictions of massive conifer mortality due to chronic temperature rise
}

\author{
N. G. McDowell, A. P. Williams, C. Xu, W. T. Pockman, L. T. Dickman, S. Sevanto, R. Pangle, J. Limousin, J. Plaut, D. S. Mackay, \\ J. Ogee, J. C. Domec, C. D. Allen, R. A. Fisher, X. Jiang, J. D. Muss, D. D. Breshears, S. A. Rauscher and C. Koven
}

Nature Climate Change 6, 295-300 (2016); published online 21 December 2015; corrected after print 7 October 2016

Additional details of the estimates of forest mortality from this Letter are required to enhance clarity and reproducibility. First, we wish to clarify what the empirically based projections shown in Figure $3 \mathrm{c}$ represent. Unlike process-based model projections that predicted percent tree mortality, our empirically derived mortality projections provide a proxy measure of the cumulative probability of a severe regional mortality event. We defined a severe regional mortality event as a year in which the regionally averaged climate of the Southwest USA (the four-corner states south of $38^{\circ} \mathrm{N}$ ) leads to an estimated predawn plant-water potential value exceeding the mortality threshold, where the estimated predawn plant water potential is based on our empirical equation that uses climate data. Similarly, the projections of mortality based on the Forest Drought Stress Index (FDSI) also represent the cumulative probability of a severe regional mortality event-where a mortality event is assumed to be a year when the FDSI (based on precipitation and vapour-pressure deficit projections in the CMIP5 RCP 8.5 scenario) is more negative than -1.41. This threshold is based on Williams, A. P. et al. (Nat. Clim. Change 3, 292-297; 2013), which found -1.41 to be the mean FDSI value during the driest half of years during Southwest USA 'megadrought' events in the 1200 s and 1500s, which were associated with widespread forest loss. Figure $3 \mathrm{c}$ indicates that the CMIP5-derived probability that such an event has occurred at some point since 1901 converges to $100 \%$ in the middle of the twenty-first century for one study species and by the end of the century for the other. These details were not sufficiently clear in our original Methods section.

In contrast, our projections of forest mortality using the process-based models of leaf predawn water potentials allowed predictions of percentage tree mortality. Although most mechanistic model projections indicate a convergence to $100 \%$ tree mortality, these models were not provided with the spatial heterogeneity in topography, soil characteristics, competition, and inter-species interactions to allow spatially explicit simulation, but rather provided a range of soil and climate conditions to bracket the full suite of conditions in Southwest USA. Through this permutation of soil properties and climate, the model outputs were used to generate tree mortality at different times over the twenty-first century, providing the step changes in mortality shown in Figure 3c (and explained in the Supplementary Information). Co-occurring substantial increases in the probability of empirically predicted mortality events and per cent tree mortality derived from the mechanistic models are complimentary in their support of one of the main conclusions of our study: that warmingdriven increase in aridity are highly likely to lead to substantial increases in Southwest USA conifer mortality throughout the twentyfirst century. As is indicated by the spread of the projections, the exact timing of this seemingly inevitable increase is highly uncertain. While it is difficult to determine the exact level of tree mortality that twenty-first century climate change will cause, the most important and realistic feature of the mortality projections shown in Figure $3 \mathrm{c}$ is the rapid increase in tree mortality in Southwest USA that may be occurring now and is likely to continue for decades to come, which is supported by multiple independent approaches. An obvious additional step that could be taken for evaluation and application of the empirical and mechanistic models is spatially explicit comparison to inventory data, which will require the use of appropriate (validated) downscaled climate data, additional ancillary data for the mechanistic models (for example, soil depth, aspect and elevation), and careful grouping of the inventory data.

Additionally, we inadvertently provided a preliminary set of equations in the Supplementary Information that were used to estimate water potential based on absolute climate values. These equations were developed during exploratory analysis and were subsequently revised to more comprehensively represent the region by using standardized climate anomalies rather than absolute climate values. The refined equations that were ultimately used were:

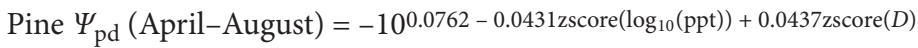

$$
\begin{aligned}
& \text { Juniper } \Psi_{\text {pd }}(\text { April-August })=-10^{0.1195-0.0814 z s c o r e}\left(\log _{10}(\mathrm{ppt})\right)+0.0606 \mathrm{zscore}(D)
\end{aligned}
$$

Here, $\Psi_{\text {pd }}$ is predawn water potential $(\mathrm{MPa})$, ppt is precipitation $(\mathrm{mm})$ total, and $D$ is mean vapour-pressure deficit $(\mathrm{kPa})$. Both variables are evaluated over the 12 months beginning in previous September and ending in the following August. The term 'zscore' indicates that these annual values of $\log _{10}(\mathrm{ppt})$ and $D$ are standardized (converted to standard deviation anomalies) relative to the temporal mean and variability of the 1961-2000 period. Units of ppt and $D$ do not matter since these values are standardized within the equations. While these equations are more refined for the region than those previously reported, either set of equations supports our general conclusion that a large increase in Southwest USA tree mortality is likely to occur by 2100. 